\title{
Agegraphic dark energy as a quintessence
}

\author{
Jingfei Zhang, a , Xin Zhang, ${ }^{2, b}$, Hongya Liu ${ }^{3, c}$ \\ ${ }^{1}$ School of Physics and Optoelectronic Technology, Dalian University of Technology, Dalian 116024, P.R. China \\ ${ }^{2}$ Kavli Institute for Theoretical Physics China, Institute of Theoretical Physics, Chinese Academy of Sciences \\ (KITPC/ITP-CAS), P.O. Box 2735, Beijing 100080, P.R. China \\ 3 School of Physics and Optoelectronic Technology, Dalian University of Technology, Dalian 116024, P.R. China
}

Received: 19 November 2007 / Revised version: 9 January 2008 /

Published online: 1 February 2008 - (C) Springer-Verlag / Società Italiana di Fisica 2008

\begin{abstract}
Recently, a dark energy model characterized by the age of the universe, dubbed "agegraphic dark energy", was proposed by Cai. In this paper, a connection between the quintessence scalar-field and the agegraphic dark energy is established, and accordingly, the potential of the agegraphic quintessence field is constructed.
\end{abstract}

\section{Introduction}

Many cosmological experiments, such as observations of large scale structure (LSS) [1-3], searches for type Ia supernovae (SNIa) $[4,5]$ and measurements of the cosmic microwave background (CMB) anisotropy [6,7], all suggest that our universe is experiencing an accelerating expansion at the present time. Following the standard FriedmannRobertson-Walker (FRW) cosmology such an expansion implies the existence of a mysterious dominant component, dark energy, with large enough negative pressure. Although we can affirm that the ultimate fate of the universe is determined by the feature of dark energy, the nature of dark energy as well as its cosmological origin remain enigmatic (for reviews, see e.g. [8-10]). It is fair to say that disclosing the nature of dark energy is one of the central problems in the research of both cosmology and theoretical physics at present. Researchers proposed many candidates to interpret or describe the properties of dark energy. The simplest candidate for dark energy is the famous cosmological constant which has the equation of state $w=-1$. However, the cosmological constant scenario, is plagued with the so-called "fine-tuning problem" and "coincidence problem" [11-23]. Theorists have made lots of efforts to try to resolve the cosmological constant problem, but all these efforts seem to be unsuccessful. Of course the theoretical consideration is still in process and has made some progresses.

An alternative proposal for dark energy is the dynamical dark energy scenario which is often realized by scalar field mechanism. It suggests that the energy form with negative pressure is provided by a scalar field evolving down a proper potential. A famous example of scalar-field dark energy is

\footnotetext{
a e-mail: jfzhang@student.dlut.edu.cn

b e-mail: zhangxin@itp.ac.cn

c e-mail: hyliu@dlut.edu.cn
}

the so-called "quintessence" [24-32]. Provided that the evolution of the field is slow enough, the kinetic energy density is less than the potential energy density, giving rise to the negative pressure responsible to the cosmic acceleration. So far, besides quintessence, a host of scalar-field dark energy models have been studied such as $K$-essence [33, 34], hessence [35-41], tachyon [42, 43], phantom [44, 45], ghost condensate [46-48] and quintom [49-51], and so forth. But we should note that the mainstream viewpoint regards the scalar field dark energy models as an effective description of an underlying theory of dark energy. In addition, other proposals on dark energy include interacting dark energy models [52-55], braneworld models [56,57], and Chaplygin gas models [58-60], etc.

However, we still can make some attempts to probe the nature of dark energy according to some principles of quantum gravity although a complete theory of quantum gravity is not available today. The holographic dark energy model [61-77] is just an appropriate and interesting example, which is constructed in the light of the holographic principle of quantum gravity theory. That is to say, the holographic dark energy model possesses some significant features of an underlying theory of dark energy. More recently, a new dark energy model, dubbed agegraphic dark energy, has been proposed [78], which takes into account the uncertainty relation of quantum mechanics together with the gravitational effect in general relativity.

In the general relativity, one can measure the spacetime without any limit of accuracy. However, in the quantum mechanics, the well-known Heisenberg uncertainty relation puts a limit of accuracy in these measurements. Following the line of quantum fluctuations of spacetime, Károlyházy and his collaborators [79-81] (see also [82]) made an interesting observation concerning the distance measurement for Minkowski spacetime through a light-clock Gedanken experiment, namely, the distance $t$ in Minkowski spacetime 
cannot be known to a better accuracy than

$$
\delta t=\lambda t_{\mathrm{p}}^{2 / 3} t^{1 / 3},
$$

where $\lambda$ is a dimensionless constant of order unity. We use the units $\hbar=c=k_{\mathrm{B}}=1$ throughout this paper. Thus, one can use the terms like length and time interchangeably, whereas $l_{\mathrm{p}}=t_{\mathrm{p}}=1 / m_{\mathrm{p}}$ with $l_{\mathrm{p}}, t_{\mathrm{p}}$ and $m_{\mathrm{p}}$ being the reduced Planck length, time and mass respectively.

The Károlyházy relation (1) together with the timeenergy uncertainty relation enables one to estimate a quantum energy density of the metric fluctuations of Minkowski spacetime $[82,83]$. Following $[82,83]$, with respect to (1) a length scale $t$ can be known with a maximum precision $\delta t$ determining thereby a minimal detectable cell $\delta t^{3} \sim t_{\mathrm{p}}^{2} t$ over a spatial region $t^{3}$. Such a cell represents a minimal detectable unit of spacetime over a given length scale $t$. If the age of the Minkowski spacetime is $t$, then over a spatial region with linear size $t$ (determining the maximal observable patch) there exists a minimal cell $\delta t^{3}$ the energy of which due to time-energy uncertainty relation can not be smaller than $[82,83]$

$$
E_{\delta t^{3}} \sim t^{-1} .
$$

Therefore, the energy density of metric fluctuations of Minkowski spacetime is given by $[82,83]$

$$
\rho_{q} \sim \frac{E_{\delta t^{3}}}{\delta t^{3}} \sim \frac{1}{t_{\mathrm{p}}^{2} t^{2}} \sim \frac{m_{\mathrm{p}}^{2}}{t^{2}}
$$

In [83] (see also [78]), it is noticed that the Károlyházy relation (1) naturally obeys the holographic black hole entropy bound. In fact, the holographic dark energy [61-63] also stems from the the idea of holographic black hole entropy bound. It is worth noting that the form of energy density (3) is similar to the one of holographic dark energy [61-63], i.e., $\rho_{\Lambda} \sim l_{\mathrm{p}}^{-2} l^{-2}$. The similarity between $\rho_{q}$ and $\rho_{\Lambda}$ might reveal some universal features of quantum gravity, although they arise from different ways. But, the agegraphic dark energy model avoids the causality problem which exists in the holographic dark energy models. For extensive studies on agegraphic dark energy, see [84-89].

By far, hundreds of dark energy models have been constructed. However, it is hard to say that we are close to the Grail of revealing the nature of dark energy (that almost means we can understand the quantum gravity well). Although going along the holographic principle of quantum gravity may provide a hopeful way towards the aim, it is hard to believe that the physical foundation of agegraphic dark energy is convincing enough. Actually, it is fair to say that almost all dynamical dark energy models are settled at the phenomenological level, neither holographic dark energy model nor agegraphic dark energy model is exception.

Though, under such circumstances, the models of holographic and agegraphic dark energy, to some extent, still have some advantage comparing to other dynamical dark energy models because at least they are built according to some fundamental principle - holographic principle - in quantum gravity. We thus may as well view that this class of models possesses some features of an underlying theory of dark energy. Now, we are interested in that if we assume the agegraphic dark energy scenario as the underlying theory of dark energy, how the low-energy effective scalar-field model can be used to describe it. In this direction, we can establish a correspondence between the agegraphic dark energy and quintessence scalar field, and describe agegraphic dark energy in this case effectively by making use of quintessence. We refer to this case as "agegraphic quintessence". The quintessence potential $V(\phi)$ can be reconstructed from supernova observational data [90-93]. In addition, from some specific parametrization forms of the equation of state $w(z)$, one can also reconstruct the quintessence potential $V(\phi)[94,95]$. The reconstruction method can also be generalized to scalar-tensor theories [96-98], $f(R)$ gravity [99], a dark energy fluid with viscosity terms [100-102], and also the generalized ghost condensate model [103]. For a reconstruction program for a very general scalar-field Lagrangian density see [104]. For the scalar-field effective description of the holographic dark energy see [105-108]. In this paper, we shall reconstruct the quintessence potential and the dynamics of the scalar field in the light of the agegraphic dark energy.

In the next section, we briefly review the original agegraphic dark energy model proposed in [78] and the new agegraphic dark energy model proposed in [87]. In Sect. 3, we establish the correspondence between the agegraphic dark energy and the quintessence. The quintessence potential and the dynamics of the scalar field are also constructed in the light of the agegraphic dark energy. Conclusion is given in Sect. 4 .

\section{Agegraphic dark energy models}

\subsection{Model 1: the original agegraphic dark energy model}

Based on the energy density (3), a so-called agegraphic dark energy model was proposed in [78]. There, as the most natural choice, the time scale $t$ in (3) is chosen to be the age of the universe

$$
T=\int_{0}^{a} \frac{\mathrm{d} a}{H a},
$$

where $a$ is the scale factor of our universe; $H \equiv \dot{a} / a$ is the Hubble parameter; a dot denotes the derivative with respect to cosmic time. Thus, the energy density of the agegraphic dark energy is given by [78]

$$
\rho_{q}=\frac{3 n^{2} m_{\mathrm{p}}^{2}}{T^{2}},
$$

where the numerical factor $3 n^{2}$ is introduced to parameterize some uncertainties, such as the species of quantum fields in the universe, the effect of curved spacetime (since the energy density is derived for Minkowski spacetime), 
and so on. Obviously, since the present age of the universe $T_{0} \sim H_{0}^{-1}$ (the subscript " 0 " indicates the present value of the corresponding quantity; we set $a_{0}=1$ ), the present energy density of the agegraphic dark energy explicitly meets the observed value naturally, provided that the numerical factor $n$ is of order unity. In addition, by choosing the age of the universe rather than the future event horizon as the length measure, the drawback concerning causality in the holographic dark energy model [61-63] does not exist in the agegraphic dark energy model [78].

If we consider a flat Friedmann-Robertson-Walker (FRW) universe containing agegraphic dark energy and pressureless matter, the corresponding Friedmann equation reads

$$
H^{2}=\frac{1}{3 m_{\mathrm{p}}^{2}}\left(\rho_{m}+\rho_{q}\right)
$$

or equivalently,

$$
E(z) \equiv \frac{H(z)}{H_{0}}=\left(\frac{\Omega_{m 0}(1+z)^{3}}{1-\Omega_{q}}\right)^{1 / 2},
$$

where $z=(1 / a)-1$ is the redshift of the universe. It is convenient to introduce the fractional energy densities $\Omega_{i} \equiv$ $\rho_{i} /\left(3 m_{\mathrm{p}}^{2} H^{2}\right)$ for $i=m$ and $q$. From (5), it is easy to find

$$
\Omega_{q}=\frac{n^{2}}{H^{2} T^{2}}
$$

whereas $\Omega_{m}=1-\Omega_{q}$ from (6). By using (5)-(8) and the energy conservation equation $\dot{\rho}_{m}+3 H \rho_{m}=0$, we obtain the equation of motion for $\Omega_{q}$ as [78]

$$
\Omega_{q}^{\prime}=\Omega_{q}\left(1-\Omega_{q}\right)\left(3-\frac{2}{n} \sqrt{\Omega_{q}}\right)
$$

where a prime denotes the derivative with respect to $N \equiv$ $\ln a$. Obviously, (9) can be rewritten as

$$
\frac{\mathrm{d} \Omega_{q}}{d z}=-(1+z)^{-1} \Omega_{q}\left(1-\Omega_{q}\right)\left(3-\frac{2}{n} \sqrt{\Omega_{q}}\right) .
$$

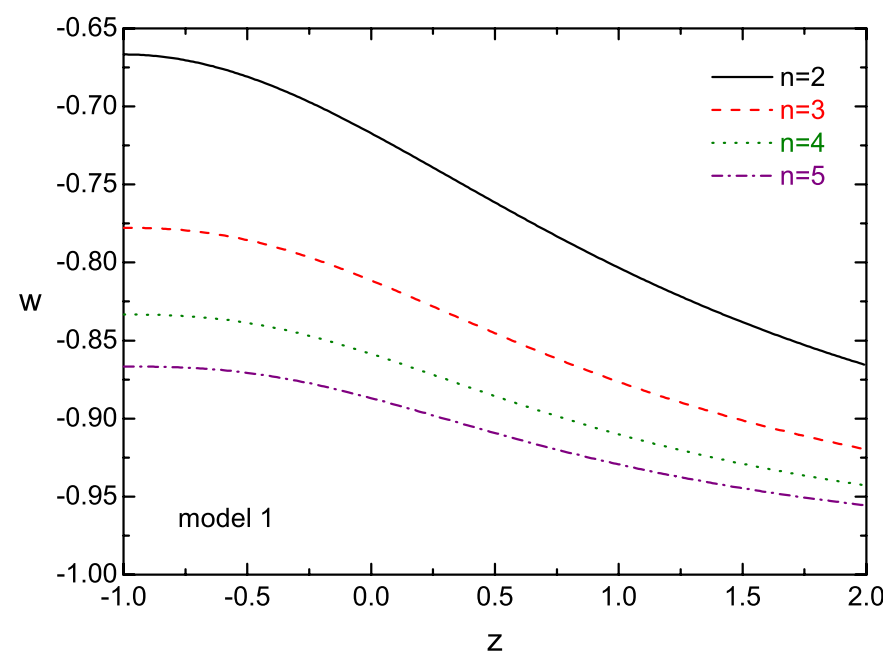

From the energy conservation equation $\dot{\rho}_{q}+3 H\left(\rho_{q}+p_{q}\right)=$ 0 , as well as (5) and (8), it is easy to find that the equationof-state parameter (EoS) of the agegraphic dark energy $w_{q} \equiv p_{q} / \rho_{q}$ is given by [78]

$$
w_{q}=-1+\frac{2}{3 n} \sqrt{\Omega_{q}} .
$$

\subsection{Model 2: the new model of agegraphic dark energy}

By choosing the time scale to be the conformal time $\eta$, Wei and Cai proposed a new agegraphic dark energy model [87], the new energy density of the agegraphic dark energy reads

$$
\rho_{q}=\frac{3 n^{2} m_{\mathrm{p}}^{2}}{\eta^{2}}
$$

where the conformal time

$$
\eta \equiv \int \frac{\mathrm{d} t}{a}=\int \frac{\mathrm{d} a}{a^{2} H} .
$$

The corresponding fractional energy density is given by

$$
\Omega_{q}=\frac{n^{2}}{H^{2} \eta^{2}} .
$$

We consider a flat FRW universe containing the new agegraphic dark energy and pressureless matter. By using (6), (12), (13) and the energy conservation equation $\dot{\rho}_{m}+3 H \rho_{m}=0$, we find that the equation of motion for $\Omega_{q}$ is given by

$$
\frac{\mathrm{d} \Omega_{q}}{\mathrm{~d} a}=\frac{\Omega_{q}}{a}\left(1-\Omega_{q}\right)\left(3-\frac{2}{n} \frac{\sqrt{\Omega_{q}}}{a}\right) .
$$

Apparently, (15) can also be rewritten as

$$
\frac{\mathrm{d} \Omega_{q}}{\mathrm{~d} z}=-\Omega_{q}\left(1-\Omega_{q}\right)\left(3(1+z)^{-1}-\frac{2}{n} \sqrt{\Omega_{q}}\right) .
$$

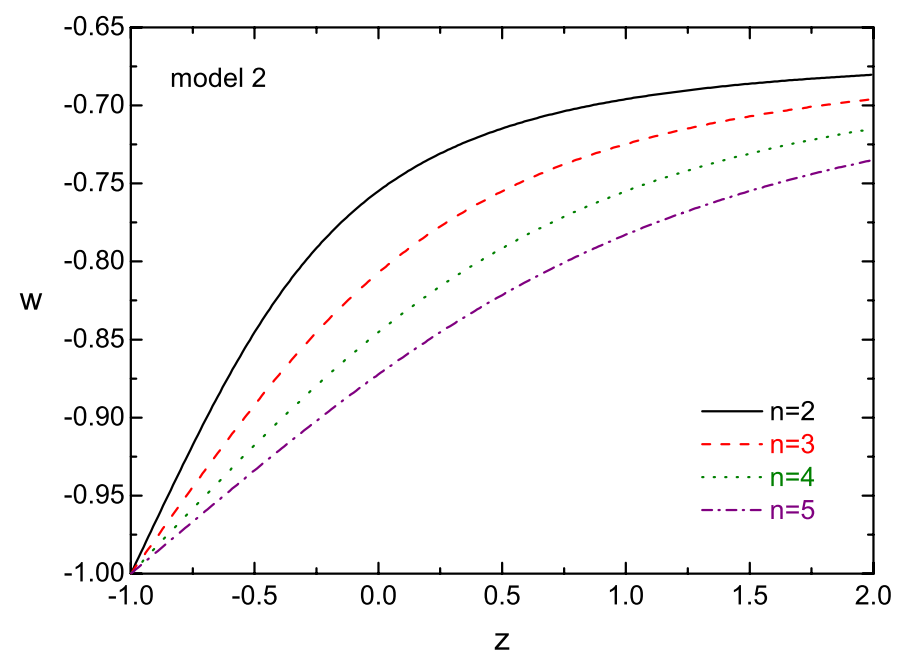

Fig. 1. The evolution of $w_{q}$ for model 1 and model 2 with different model parameter $n$ 
From the energy conservation equation $\dot{\rho}_{q}+3 H\left(\rho_{q}+p_{q}\right)=$ 0 , as well as (12) and (14), it is easy to find that the EoS of the new agegraphic dark energy, $w_{q} \equiv p_{q} / \rho_{q}$, is given by

$$
w_{q}=-1+\frac{2}{3 n}(1+z) \sqrt{\Omega_{q}} .
$$

In order to compare the behaviors of the two agegraphic dark energy models, we plot the evolution curves of $w$ in Fig. 1 for the two models with different parameter $n$. It is easy to see that the behavior of the new agegraphic dark energy is very different from the original agegraphic energy model. For model 1, the EoS has a "thawing" feature. At earlier time where $\Omega_{q} \rightarrow 0$, one has $w_{q} \rightarrow-1$. Namely, the dark energy behaves like a cosmological constant at earlier time. At later time where $\Omega_{q} \rightarrow 1$, the EoS goes to $w_{q}=-1+\frac{2}{3 n}$. Therefore the fate of our universe is a forever accelerated expansion with a power-law form in this dark energy model [78]. While for model 2 , the EoS has a "freezing" feature, i.e., in the late time $w_{q} \rightarrow-1$ when $a \rightarrow \infty$, it mimics a cosmological constant regardless of the value of $n$.

\section{Reconstructing agegraphic quintessence}

In this section, we will construct the agegraphic quintessence models, connecting the quintessence scalar-field with the agegraphic dark energy.

The quintessence scalar field $\phi$ evolves in its potential $V(\phi)$ and seeks to roll towards the minimum of the potential, according to the Klein-Gordon equation $\ddot{\phi}+$ $3 H \dot{\phi}=-\mathrm{d} V / \mathrm{d} \phi$. The rate of evolution is driven by the slope of the potential and damped by the cosmic expansion through the Hubble parameter $H$. The energy density and pressure are $\rho_{\phi}=\dot{\phi}^{2} / 2+V, p_{\phi}=\dot{\phi}^{2} / 2-V$, so that the equation of state of quintessence $w_{\phi}=p_{\phi} / \rho_{\phi}$ evolves in a region of $-1<w_{\phi}<1$. Usually, for making the universe's expansion accelerate, it should be required that $w_{\phi}$ must satisfy $w_{\phi}<-1 / 3$. Obviously, both model 1 and model 2 can be described by the quintessence. We establish a correspondence between the agegraphic dark energy and quintessence scalar field, and describe agegraphic dark energy effectively by making use of quintessence. We refer to this case as "agegraphic quintessence".

Now we are focussing on the reconstruction of the agegraphic quintessence. We shall reconstruct the quintessence potential and the dynamics of the scalar field in the light of the agegraphic dark energy with $n \geq 1$. According to the forms of quintessence energy density and pressure, one can easily derive the scalar potential and kinetic energy term as

$$
\begin{aligned}
\frac{V(\phi)}{\rho_{\mathrm{c} 0}} & =\frac{1}{2}\left(1-w_{\phi}\right) \Omega_{\phi} E^{2}, \\
\frac{\dot{\phi}^{2}}{\rho_{\mathrm{c} 0}} & =\left(1+w_{\phi}\right) \Omega_{\phi} E^{2},
\end{aligned}
$$

where $\rho_{\mathrm{c} 0}=3 m_{\mathrm{p}}^{2} H_{0}^{2}$ is today's critical density of the universe. If we establish the correspondence between the agegraphic dark energy with $n \geq 1$ and quintessence scalar field, then $E, \Omega_{\phi}$ and $w_{\phi}$ are given by (7), (10) and (11) for model 1 , while by (7), (16) and (17) for model 2. Furthermore, the derivative of the scalar field $\phi$ with respect to the redshift $z$ can be given by

$$
\frac{\mathrm{d} \phi}{\mathrm{d} z}= \pm \frac{m_{\mathrm{p}} \sqrt{3\left(1+w_{\phi}\right) \Omega_{\phi}}}{1+z}
$$

where the sign is actually arbitrary since it can be changed by a redefinition of the field, $\phi \rightarrow-\phi$. Consequently, we can easily obtain the evolutionary form of the field

$$
\phi(z)=\int_{0}^{z} \frac{\mathrm{d} \phi}{\mathrm{d} z} \mathrm{~d} z,
$$

by fixing the field amplitude at the present epoch $(z=0)$ to be zero, $\phi(0)=0$.

The reconstructed quintessence potential $V(\phi)$ is plotted in Fig. 2, where $\phi(z)$ is also reconstructed according
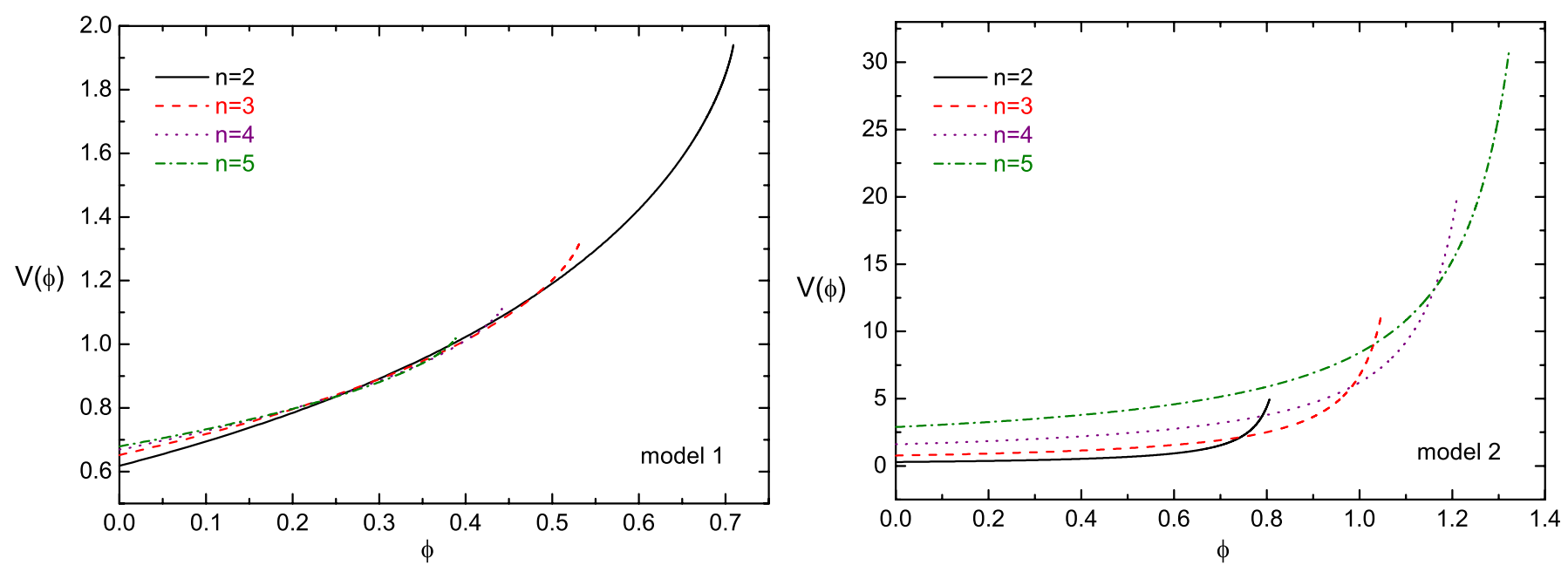

Fig. 2. The reconstruction of the potential for the agegraphic quintessence where $\phi$ is in unit of $m_{\mathrm{p}}$ and $V(\phi)$ in $\rho_{\mathrm{c} 0}$. We take here $\Omega_{m 0}=0.28$ 

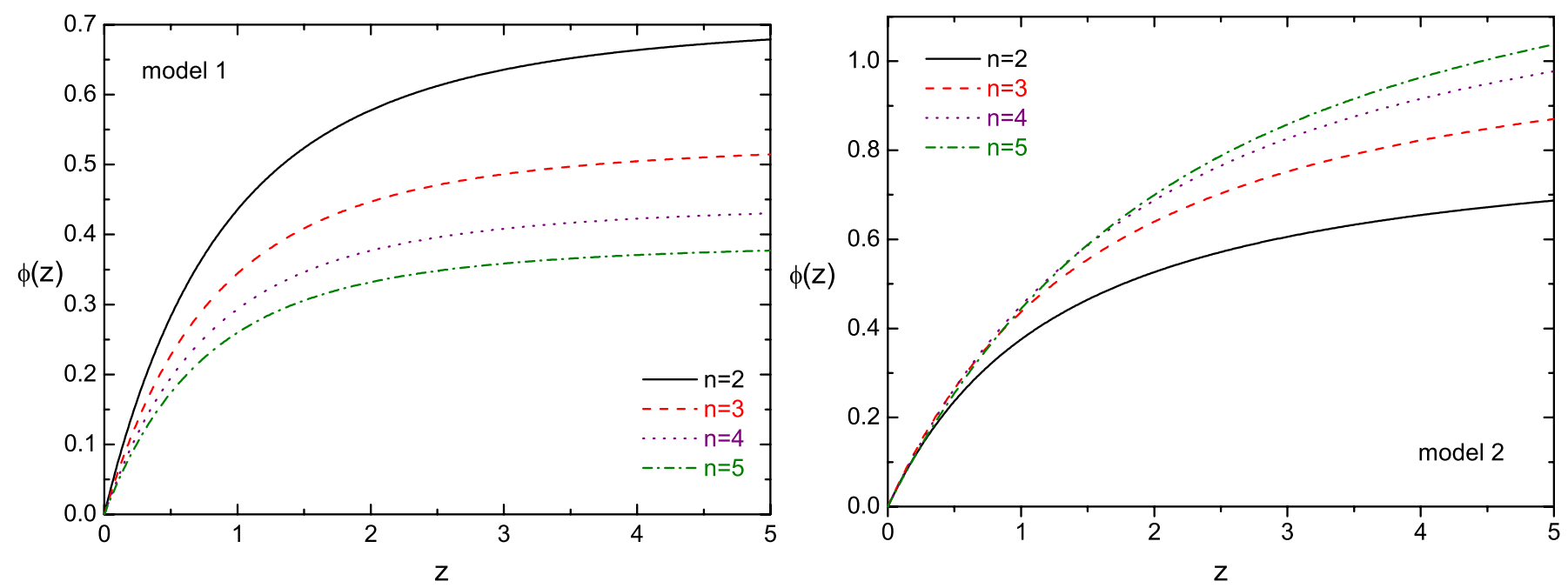

Fig. 3. The revolution of the scalar-field $\phi(z)$ for the agegraphic quintessence, where $\phi$ is in unit of $m_{\mathrm{p}}$. We take here $\Omega_{m 0}=0.28$

to (20) and (21), also displayed in Fig. 3. Selected curves are plotted for the cases of $n=2,3,4$ and 5 , and the present fractional matter density is chosen to be $\Omega_{m 0}=$ 0.28 . From Figs. 2 and 3, we can see the dynamics of the scalar field explicitly. As suggested in [109], quintessence models can be divided into two classes, "thawing" models and "freezing" models. Thawing models depict those scalar fields that evolve from $w=-1$ but grow less negative with time as $\mathrm{d} w / \mathrm{d} \ln a>0$; freezing models, whereas, describe those fields evolve from $w>-1, \mathrm{~d} w / \mathrm{d} \ln a<0$ to $w \rightarrow-1$, $\mathrm{d} w / \mathrm{d} \ln a \rightarrow 0$. Roughly, the agegraphic quintessence corresponding to model 1 should be ascribed to the "thawing" model, while the agegraphic quintessence corresponding to model 2 should be ascribed to "freezing" model. As we have seen, the dynamics of the agegraphic quintessence can be explored explicitly by the reconstruction.

\section{Conclusion}

In conclusion, we suggest in this paper a correspondence between the agegraphic dark energy scenario and the quintessence scalar-field model. We adopt the viewpoint that the scalar field models of dark energy are effective theories of an underlying theory of dark energy. A new dark energy model, named as "agegraphic dark energy", has been proposed by Cai [78], based on the Károlyházy uncertainty relation, which arises from the quantum mechanics together with general relativity. Perhaps, one may argue that the agegraphic dark energy lacks convincing physical foundation and the two models with energy densities in (5) and (12) considered in this paper are just two types of the phenomenologically parametrized equation of state of dark energy. However, anyway, one should admit that it is an interesting attempt to consider the nature of dark energy based on some combination of quantum mechanics and general relativity.

If we regard the scalar-field model (such as quintessence) as an effective description of such a theory, we should be capable of using the scalar-field model to mimic the evolving behavior of the agegraphic dark energy and reconstructing this scalar-field model according to the evolutionary behavior of agegraphic dark energy. We show that the agegraphic dark energy with $n \geq 1$ can be described totally by the quintessence in a certain way. A correspondence between the agegraphic dark energy and quintessence has been established, and the potential of the agegraphic quintessence and the dynamics of the field have been reconstructed.

Acknowledgements. This work was supported partially by grants from the National Natural Science Foundation of China (No. 10573003 and No. 10705041), the China Postdoctoral Science Foundation (No. 20060400104), the K.C. Wong Education Foundation and the National Basic Research Program of China (No. 2003CB716300).

\section{References}

1. SDSS Collaboration, M. Tegmark et al., Phys. Rev. D 69, 103501 (2004) [astro-ph/0310723]

2. SDSS Collaboration, K. Abazajian et al., Astron. J. 128, 502 (2004) [astro-ph/0403325]

3. SDSS Collaboration, K. Abazajian et al., Astron. J. 129, 1755 (2005) [astro-ph/0410239]

4. Supernova Search Team Collaboration, A.G. Riess et al., Astron. J. 116, 1009 (1998) [astro-ph/9805201]

5. Supernova Cosmology Project Collaboration, S. Perlmutter et al., Astrophys. J. 517, 565 (1999) [astro-ph/9812133]

6. WMAP Collaboration, D.N. Spergel et al., Astrophys. J. Suppl. 148, 175 (2003) [astro-ph/0302209]

7. D.N. Spergel et al., astro-ph/0603449

8. S. Weinberg, Rev. Mod. Phys. 61, 1 (1989)

9. S.M. Carroll, Living Rev. Relat. 4, 1 (2001) [arXiv:astro$\mathrm{ph} / 0004075$ ]

10. P.J.E. Peebles, B. Ratra, Rev. Mod. Phys. 75, 559 (2003) [arXiv:astro-ph/0207347]

11. T. Padmanabhan, Phys. Rep. 380, 235 (2003) [hepth/0212290] 
12. S.M. Carroll, astro-ph/0310342

13. R. Bean, S. Carroll, M. Trodden, astro-ph/0510059

14. V. Sahni, A.A. Starobinsky, Int. J. Mod. Phys. D 9, 373 (2000) [astro-ph/9904398]

15. T. Padmanabhan, Curr. Sci. 88, 1057 (2005) [astro$\mathrm{ph} / 0411044]$

16. S. Nobbenhuis, Found. Phys. 36, 613 (2006) [gr-qc/ 0411093]

17. E.J. Copeland, M. Sami, S. Tsujikawa, Int. J. Mod. Phys. D 15, 1753 (2006) [hep-th/0603057]

18. A. Albrecht et al., astro-ph/0609591

19. R. Trotta, R. Bower, astro-ph/0607066

20. M. Kamionkowski, arXiv:astro-ph/0706.2986

21. B. Ratra, M.S. Vogeley, arXiv:astro-ph/0706.1565

22. E.V. Linder, arXiv:astro-ph/0705.4102

23. P.J. Steinhardt, in Critical Problems in Physics, ed. by V.L. Fitch, D.R. Marlow (Princeton University Press, Princeton, NJ, 1997)

24. P.J.E. Peebles, B. Ratra, Astrophys. J. 325, L17 (1988)

25. B. Ratra, P.J.E. Peebles, Phys. Rev. D 37, 3406 (1988)

26. C. Wetterich, Nucl. Phys. B 302, 668 (1988)

27. J.A. Frieman, C.T. Hill, A. Stebbins, I. Waga, Phys. Rev. Lett. 75, 2077 (1995) [astro-ph/9505060]

28. M.S. Turner, M.J. White, Phys. Rev. D 56, 4439 (1997) [astro-ph/9701138]

29. R.R. Caldwell, R. Dave, P.J. Steinhardt, Phys. Rev. Lett. 80, 1582 (1998) [astro-ph/9708069]

30. A.R. Liddle, R.J. Scherrer, Phys. Rev. D 59, 023509 (1999) [astro-ph/9809272]

31. I. Zlatev, L.M. Wang, P.J. Steinhardt, Phys. Rev. Lett. 82, 896 (1999) [astro-ph/9807002]

32. P.J. Steinhardt, L.M. Wang, I. Zlatev, Phys. Rev. D 59, 123504 (1999) [astro-ph/9812313]

33. C. Armendariz-Picon, V.F. Mukhanov, P.J. Steinhardt, Phys. Rev. Lett. 85, 4438 (2000) [astro-ph/0004134]

34. C. Armendariz-Picon, V.F. Mukhanov, P.J. Steinhardt, Phys. Rev. D 63, 103510 (2001) [astro-ph/0006373]

35. H. Wei, R.G. Cai, D.F. Zeng, Class. Quantum Grav. 22, 3189 (2005) [hep-th/0501160]

36. H. Wei, R.G. Cai, Phys. Rev. D 72, 123507 (2005) [astro$\mathrm{ph} / 0509328]$

37. M. Alimohammadi, H. Mohseni Sadjadi, Phys. Rev. D 73, 083527 (2006) [hep-th/0602268]

38. W. Zhao, Y. Zhang, Phys. Rev. D 73, 123509 (2006) [astro-ph/0604460]

39. H. Wei, N.N. Tang, S.N. Zhang, Phys. Rev. D 75, 043009 (2007) [astro-ph/0612746]

40. H. Wei, S.N. Zhang, arXiv:gr-qc/0705.4002

41. W. Zhao, arXiv:astro-ph/0706.2211

42. A. Sen, JHEP 0207, 065 (2002) [hep-th/0203265]

43. T. Padmanabhan, Phys. Rev. D 66, 021301 (2002) [hepth/0204150]

44. R.R. Caldwell, Phys. Lett. B 545, 23 (2002) [astro$\mathrm{ph} / 9908168]$

45. R.R. Caldwell, M. Kamionkowski, N.N. Weinberg, Phys. Rev. Lett. 91, 071301 (2003) [astro-ph/0302506]

46. N. Arkani-Hamed, H.C. Cheng, M.A. Luty, S. Mukohyama, JHEP 0405, 074 (2004) [hep-th/0312099]

47. S. Mukohyama, JCAP 0610, 011 (2006) [hep-th/0607181]

48. F. Piazza, S. Tsujikawa, JCAP 0407, 004 (2004) [hepth/0405054]

49. B. Feng, X.L. Wang, X.M. Zhang, Phys. Lett. B 607, 35 (2005) [astro-ph/0404224]
50. Z.K. Guo, Y.S. Piao, X.M. Zhang, Y.Z. Zhang, Phys. Lett. B 608, 177 (2005) [astro-ph/0410654]

51. X. Zhang, Commun. Theor. Phys. 44, 762 (2005)

52. L. Amendola, Phys. Rev. D 62, 043511 (2000) [astro$\mathrm{ph} / 9908023]$

53. D. Comelli, M. Pietroni, A. Riotto, Phys. Lett. B 571, 115 (2003) [hep-ph/0302080]

54. X. Zhang, Mod. Phys. Lett. A 20, 2575 (2005) [astro$\mathrm{ph} / 0503072]$

55. X. Zhang, Phys. Lett. B 611, 1 (2005) [astro-ph/0503075]

56. C. Deffayet, G.R. Dvali, G. Gabadadze, Phys. Rev. D 65, 044023 (2002) [astro-ph/0105068]

57. V. Sahni, Y. Shtanov, JCAP 0311, 014 (2003) [astro$\mathrm{ph} / 0202346]$

58. A.Y. Kamenshchik, U. Moschella, V. Pasquier, Phys. Lett. B 511, 265 (2001) [gr-qc/0103004]

59. M.C. Bento, O. Bertolami, A.A. Sen, Phys. Rev. D 66, 043507 (2002) [gr-qc/0202064]

60. X. Zhang, F.Q. Wu, J. Zhang, JCAP 0601, 003 (2006) [astro-ph/0411221]

61. M. Li, Phys. Lett. B 603, 1 (2004) [hep-th/0403127]

62. Q.G. Huang, M. Li, JCAP 0408, 013 (2004) [astro$\mathrm{ph} / 0404229]$

63. J. Zhang, X. Zhang, H. Liu, Eur. Phys. J. C 52, 693 (2007) [arXiv:hep-th/0708.3121]

64. Q.G. Huang, Y.G. Gong, JCAP 0408, 006 (2004) [astro$\mathrm{ph} / 0403590]$

65. X. Zhang, F.Q. Wu, Phys. Rev. D 72, 043524 (2005) [astro-ph/0506310]

66. Z. Chang, F.Q. Wu, X. Zhang, Phys. Lett. B 633, 14 (2006) [astro-ph/0509531]

67. X. Zhang, F.Q. Wu, Phys. Rev. D 76, 023502 (2007) [astro-ph/0701405]

68. H. Wei, S.N. Zhang, Phys. Rev. D 76, 063003 (2007) [arXiv:astro-ph/0707.2129]

69. A.G. Cohen, D.B. Kaplan, A.E. Nelson, Phys. Rev. Lett. 82, 4971 (1999) [hep-th/9803132]

70. S.D.H. Hsu, Phys. Lett. B 594, 13 (2004) [hep-th/ 0403052]

71. P. Horava, D. Minic, Phys. Rev. Lett. 85, 1610 (2000) [hepth/0001145]

72. S.D. Thomas, Phys. Rev. Lett. 89, 081301 (2002)

73. Q.G. Huang, M. Li, JCAP 0503, 001 (2005) [hep-th/ 0410095]

74. X. Zhang, Int. J. Mod. Phys. D 14, 1597 (2005) [astro$\mathrm{ph} / 0504586]$

75. M.R. Setare, J. Zhang, X. Zhang, JCAP 0703, 007 (2007) [gr-qc/0611084]

76. J. Zhang, X. Zhang, H. Liu, Phys. Lett. B 659, 26 (2008) [arXiv:astro-ph/0705.4145]

77. B. Chen, M. Li, Y. Wang, Nucl. Phys. B 774, 256 (2007) [astro-ph/0611623]

78. R.G. Cai, Phys. Lett. B 657, 228 (2007) [arXiv:hepth/0707.4049]

79. F. Károlyházy, Nuovo Cim. A 42, 390 (1966)

80. F. Károlyházy, A. Frenkel, B. Lukács, in Physics as Natural Philosophy, ed. by A. Simony, H. Feschbach (MIT Press, Cambridge, MA, 1982)

81. F. Károlyházy, A. Frenkel, B. Lukács, in Quantum Concepts in Space and Time, ed. by R. Penrose, C.J. Isham (Clarendon Press, Oxford, 1986)

82. M. Maziashvili, Int. J. Mod. Phys. D 16, 1531 (2007) [arXiv:gr-qc/0612110] 
83. M. Maziashvili, Phys. Lett. B 652, 165 (2007) [arXiv:grqc/0705.0924]

84. H. Wei, R.G. Cai, arXiv:hep-th/0707.4052

85. H. Wei, R.G. Cai, Phys. Lett. B 655, 1 (2007) [arXiv:grqc/0707.4526]

86. X. Wu, Y. Zhang, H. Li, R.G. Cai, Z.H. Zhu, arXiv:astroph/0708.0349

87. H. Wei, R.G. Cai, arXiv:astro-ph/0708.0884

88. Y. Zhang, H. Li, X. Wu, H. Wei, R.G. Cai, arXiv:astroph/0708.1214

89. H. Wei, R.G. Cai, arXiv:astro-ph/0708.1894

90. T.D. Saini, S. Raychaudhury, V. Sahni, A.A. Starobinsky, Phys. Rev. Lett. 85, 1162 (2000) [astro-ph/9910231]

91. A.A. Starobinsky, JETP Lett. 68, 757 (1998) [Pis'ma Zh. Eksp.Teor. Fiz. 68, 721 (1998)] [astro-ph/9810431]

92. D. Huterer, M.S. Turner, Phys. Rev. D 60, 081301 (1999)[astro-ph/9808133]

93. T. Nakamura, T. Chiba, Mon. Not. R. Astron. Soc. 306, 696 (1999) [astro-ph/9810447]

94. Z.K. Guo, N. Ohta, Y.Z. Zhang, Phys. Rev. D 72, 023504 (2005) [astro-ph/0505253]

95. Z.K. Guo, N. Ohta, Y.Z. Zhang, astro-ph/0603109

96. B. Boisseau, G. Esposito-Farese, D. Polarski, A.A. Starobinsky, Phys. Rev. Lett. 85, 2236 (2000) [grqc/0001066]
97. G. Esposito-Farese, D. Polarski, Phys. Rev. D 63, 063504 (2001) [gr-qc/0009034]

98. L. Perivolaropoulos, JCAP 0510, 001 (2005)[astro-ph/ 0504582]

99. S. Capozziello, V.F. Cardone, A. Troisi, Phys. Rev. D 71, 043503 (2005) [astro-ph/0501426]

100. S. Capozziello, V.F. Cardone, E. Elizalde, S. Nojiri, S.D. Odintsov, Phys. Rev. D 73, 043512 (2006) [astro$\mathrm{ph} / 0508350]$

101. I. Brevik, O. Gorbunova, Gen. Relat. Grav. 37, 2039 (2005) [gr-qc/0504001]

102. S. Nojiri, S.D. Odintsov, Phys. Rev. D 72, 023003 (2005) [hep-th/0505215]

103. J. Zhang, X. Zhang, H. Liu, astro-ph/0612642

104. S. Tsujikawa, Phys. Rev. D 72, 083512 (2005) [astro$\mathrm{ph} / 0508542]$

105. X. Zhang, Phys. Lett. B 648, 1 (2007) [astro-ph/ 0604484]

106. X. Zhang, Phys. Rev. D 74, 103505 (2006) [astro$\mathrm{ph} / 0609699]$

107. J. Zhang, X. Zhang, H. Liu, Phys. Lett. B 651, 84 (2007) [arXiv:astro-ph/0706.1185]

108. Y.Z. Ma, X. Zhang, arXiv:astro-ph/0709.1517

109. R.R. Caldwell, E.V. Linder, Phys. Rev. Lett. 95, 141301 (2005) [astro-ph/0505494] 